\title{
Special section: Crystallography and properties of metal-organic framework compounds
}

Research on metal-organic framework (MOF) materials has been a fast-growing field over the past decade. The interest and excitement in these materials are evidenced by the explosion in the number of highly cited MOF-related papers published in recent years. The increasing attention is partly owing to the wide potential applications, including gas sorption, gas storage, gas separation, catalysis, sensors, drug delivery, imaging, electronic devices, and environmental sustainability. The development and fundamental understanding of the properties of MOF materials are therefore important to technological needs, and they are at the forefront of materials research.

Powder Diffraction has started publishing special journal sections that has and will continue to focus on the latest developments in the cross-disciplinary fields of structure science and MOF materials. The papers are published in the special sections throughout the four issues of the 2019 and the first issue of the 2020 Powder Diffraction journal.

In this December issue of Powder Diffraction, we included two technical papers. The paper "Recent Advances in Experimental Thermodynamics of Metal-Organic Frameworks" by H. Sun and D. Wu is an invited review paper that highlights the complex interplays among interrelated compositional, chemical, and topological (structural) factors in the determination of the thermodynamic parameters of MOFs. The paper "Topology of Voids and Channels in
Selected Porphyrinic Compounds" by L.P. Cook et al. describes an initial survey and the calculation of the voids and channels in a random subset of 1000 porphyrinic compounds. They discuss the characteristic of four representative compounds in further details including technological implications. The guest editors anticipate that these papers will serve the community as the reference for modern measurement capabilities, data analysis, and materials insight.

\section{Guest Editors:}

Dr. Craig Brown is a Fellow at the National Institute of Standards and Technology (NIST) where he leads the Structure and Dynamics of Materials Team that supports a suite of neutron instruments in the NIST Center for Neutron Research (NCNR). He is also an Affiliated Assistant Professor in the Chemical Engineering Department at the University of Delaware.

Dr. Winnie Wong-Ng is currently a research chemist (co-project leader) in the Materials for Energy and Sustainable Development Group of the Materials Measurement Science Division of NIST. She is also an adjunct professor at the Materials Science and Engineering Department of Boise State University.

Winnie Wong-Ng

E-mail: winnie.wong-ng@ nist.gov 